The Journal of Nonlinear $\mathbf{S}_{\text {cience and Aplications }}$ http://www.tjnsa.com

\title{
AN EFFICIENT APPLICATIONS OF HE'S VARIATIONAL ITERATION METHOD BASED ON A RELIABLE MODIFICATION OF ADOMIAN ALGORITHM FOR NONLINEAR BOUNDARY VALUE PROBLEMS
}

\author{
A. GOLBABAI *, K. SAYEVAND
}

\begin{abstract}
In this paper, the He's variational iteration method (VIM) based on a reliable modification of Adomian algorithm has been used to obtain solutions of the nonlinear boundary value problems (BVP). Comparison of the result obtained by the present method with that obtained by Adomian method [A. M. Wazwaz, Found Phys. Lett. 13 (2000) 493 and G. L. Liu, Modern Mathematical and Mechanics, (1995) 643 ] reveals that the present method is very effective and convenient.
\end{abstract}

\section{INTRODUCTION}

Recently much attention has been given to develop some analytical methods for solving BVP [4, 13]. It is well known that VIM provide the most versatile tools available in nonlinear analysis problems [1]-[18]. In this paper, we developed the VIM based on a reliable modification of Adomian algorithm for solving nonlinear boundary value problems.

The paper has been organized as follows. Section 2 gives analysis of methods. Reliable modification of Adomian algorithm for calculating nonlinear part in VIM is presented in Section 3. In Section 4 application of the approach is considered for the special type of BVP equation. Discussion and conclusion are presented in Section 5.

Date: Revised: 25 Jan. 2010.

* Corresponding author.

2000 Mathematics Subject Classification. Primary 37K40; Secondary 76B25.

Key words and phrases. Adomian polynomials, Boundary value problems, Variational iteration method. 


\section{AnAlysis OF THE METHOD}

For the purpose of illustration of the methodology to the proposed method, using variational iteration method, we begin by considering a differential equation in the formal form,

$$
L \xi+N \xi=\vartheta(x, t)
$$

where $L$ is a linear operator, $N$ a nonlinear operator and $\vartheta(x, t)$ is the source inhomogeneous term. According to the variational iteration method, we can construct a correction functional as follow

$$
\xi_{n+1}(x, t)=\xi_{n}(x, t)+\int_{0}^{t} \lambda(\xi)\left(L \xi_{n}(\xi)+N \tilde{\xi}(\xi)-\vartheta(\xi)\right) d \xi, \quad n \geq 0
$$

where $\lambda$ is a general Lagrangian multiplier [11], which can be identified optimally via the variational theory, the subscript $n$ denotes the $n$th order approximation, $\tilde{\xi}_{n}$ is considered as a restricted variation [7], 9] and [11] i.e., $\delta \tilde{\xi}_{n}=0$. So, we first determine the Lagrange multiplier $\lambda$ that will be identified optimally via integration by parts. The successive approximations $\xi_{n+1}(x, t), n \geq 0$ of the solution $\xi(x, t)$ will be readily obtained upon using the obtained Lagrange multiplier and by using any selective function $\xi_{0}$. Consequently, the solution

$$
\xi(x, t)=\lim _{n \longrightarrow \infty} \xi_{n}(x, t) .
$$

\section{Reliable modificAtion of Adomian Algorithm For CALCUlating NONLINEAR PART IN VIM}

To determine $N\left(\xi_{0}, \xi_{1}, \cdots, \xi_{j}, t\right)$, we use

$$
F(\xi(x, t))=N\left(\xi_{0}, \xi_{1}, \cdots, \xi_{j}, t\right)=\sum_{n=0}^{\infty} A_{n} .
$$

By the Wazwaz'reliable modification of Adomian algorithm we have

$$
\begin{aligned}
A_{0} & =F\left(\xi_{0}\right), \\
A_{1} & =\xi_{1} F^{\prime}\left(\xi_{0}\right), \\
A_{2} & =\xi_{2} F^{\prime}\left(\xi_{0}\right)+\frac{1}{2 !} \xi_{1}^{2} F^{\prime \prime}\left(\xi_{0}\right), \\
A_{3} & =\xi_{3} F^{\prime}\left(\xi_{0}\right)+\xi_{1} \xi_{2} F^{\prime \prime}\left(\xi_{0}\right)+\frac{1}{3 !} F^{\prime \prime \prime}\left(\xi_{0}\right), \\
A_{4} & =\xi_{4} F^{\prime}\left(\xi_{0}\right)+\left(\frac{1}{2 !} \xi_{2}^{2}+\xi_{1} \xi_{3}\right) F^{\prime \prime}\left(\xi_{0}\right)+\frac{1}{2 !} \xi_{1}^{2} \xi_{2} F^{\prime \prime \prime}\left(\xi_{0}\right)+\frac{1}{4 !} \xi_{1}^{4} F^{(i v)}\left(\xi_{0}\right), \\
& \vdots
\end{aligned}
$$

This fact is based on a reliable modification of Adomian's algorithm [15].

\section{Applying Vim for nOnlinear BVP}

Recently Adomian method [3] has been widely applied to nonlinear problems. Liu [16] and He [10] found that Adomian method could not always satisfy its boundary condition, leading to an error at its boundaries. In this paper, we 
develop the VIM based on a reliable modification of Adomian algorithm for solving nonlinear boundary value problems. This method is easy to implement and is more efficient than the Adomian method, also the lacks which mentioned in above there is not in this approach. Furthermore one famous example which was solved before by Liu and Wazwaz [15, 16] is given to illustrate the performance of the method. Consider a specific non linear PDE [15, 16]

$$
\nabla^{2} \xi+\left(\frac{\partial \xi}{\partial y}\right)^{2}=2 y+x^{4}
$$

Subject to boundary conditions

$$
\begin{array}{cl}
\xi(0, y)=0, & \xi(1, y)=y+c \\
\xi(x, 0)=c x, & \xi(x, y)=x(x+c),
\end{array}
$$

where $c$ is a constant. Liu [16 obtained the following approximate solution by Adomian method:

$$
\xi(x, y)=x(x y+c)+\frac{1}{2}\left[y(y-1)\left(\frac{x^{4}}{2}+\frac{y+1}{3}\right)+\frac{x}{30}\left(x^{5}-1\right)\right],
$$

It is obvious that the obtained Solution does not Satisfy the given boundary condition. Liu [16] suggested a weighted residual method and obtained the following approximation

$$
\xi(x, y)=x(x y+c)+\frac{1}{4} x y(y-1)\left(1-x^{3}\right),
$$

which satisfies all boundary conditions. It's clear that the accuracy of this method depends upon the choice of weighted factors. There for we present a simple but powerful VIM to the discussed problem. Now for applying VIM, first we rewrite Eq. (4.1) in the following form

$$
L_{x x}(\xi)+L_{y y}(\xi)+N(\xi)-2 y-x^{4}=0,
$$

where the notations $N(\xi)=\left(\frac{\partial \xi_{n}(s, y)}{\partial y}\right)^{2}$ symbolize the nonlinear term and the notation $L_{x x}=\frac{\partial^{2}}{\partial x^{2}}$ symbolize the linear differential operator. The correction functional for Eq.(4.4) reads

$$
\xi_{n+1}(x, t)=\xi_{n}(x, t)+\int_{0}^{t} \lambda(\xi)\left[\frac{\partial^{2}}{\partial \xi^{2}}\left(\xi_{n}\right)+L_{y y}(\tilde{\xi})+N(\tilde{\xi})-2 y-x^{4}\right] d \xi,
$$

where $n \geq 0$. Here, the linear term $L_{y y}(\xi)$ is also considered as a restricted variation in order to simplify the procedures for evaluation of solutions. Taking variation with respect to the independent variable $u_{n}$, noticing that $\delta\left(L_{y y}(\tilde{\xi})+\right.$ $\left.N\left(\tilde{\xi}_{n}\right)\right)=0$

$$
\begin{aligned}
\delta \xi_{n+1}(x, t) & =\delta \xi_{n}(x, t)+\delta \int_{0}^{t} \lambda(u)\left[\frac{\partial^{2}}{\partial u^{2}}\left(\xi_{n}\right)+L_{y y}(\tilde{\xi})+N(\tilde{\xi})-2 y-x^{4}\right] d u \\
& =\delta \xi_{n}(x, t)+\left.\lambda \delta \frac{\partial \xi_{n}}{\partial u}\right|_{u=t}-\left.\lambda^{\prime} \delta \xi_{n}\right|_{u=t}+\int_{0}^{t} \lambda^{\prime \prime}(u) \delta \xi_{n} d u=0,
\end{aligned}
$$


This yields the stationary conditions

$$
\begin{aligned}
& \lambda^{\prime \prime}(u)=0, \\
& \left.\lambda(u)\right|_{u=t}=0, \\
& 1-\left.\lambda^{\prime}(u)\right|_{u=t}=0 .
\end{aligned}
$$

This in turn gives $\lambda(u)=u-t$. Substituting this value of the Lagrange multiplier into the functional (4.5) gives the iteration formula

$$
\begin{aligned}
\xi_{n+1}(x, t)=\xi_{n}(x, t)+\int_{0}^{t}(w & -x)\left[\frac{\partial^{2} \xi_{n}(s, y)}{\partial s^{2}}+\frac{\partial^{2} \xi_{n}(s, y)}{\partial y^{2}}\right. \\
& \left.+\left(\frac{\partial \xi_{n}(s, y)}{\partial y}\right)^{2}-2 y-s^{4}\right] d s
\end{aligned}
$$

We start with an initial approximation: $\xi_{0}(x, t)=\xi(x, 0)=c x$, by the above iteration formula, we can obtain successively its approximations:

$$
\xi_{1}(x, y)=c x+x^{2} y
$$

and

$$
\xi_{2}(x, y)=\xi_{3}(x, y)=\cdots=\xi_{n}(x, y)=c x+x^{2} y .
$$

Finally, we approximate the solution $\xi(x, t)=\lim _{n \longrightarrow \infty} \xi_{n}(x, t)$. Thus, we obtain

$$
\xi(x, y)=c x+x^{2} y
$$

which is an exact solution.

\section{Remark:}

- Equation(1)has been solved by Liu [16] using Adomian decomposition method. Liu obtained approximate solution, but we obtain exact solution after 2 iteration.

- Wazwaz [15]applied Adomian method and obtained an infinite series which convergence to the exact solution. Compared with the [15], the present method has some obvious merits,for example the method is very straightforward, and the solution procedure can be done easily.

\section{Discussion AND CONCLUSION}

The variational iteration method is a powerful tool which is capable of handling linear/nonlinear partial differential equations. The method has been successfully applied to nonlinear boundary value problems. Also, comparisons were made between He's variational iteration method and Adomian decomposition method (ADM). For nonlinear equations that arise frequently to express nonlinear phenomenon, He's variational iteration method gives the solution rapidly if compared with Adomian method. In this paper, the He's variational iterative method based on a reliable of Adomian'algorithm has been successfully applied to the famous case of non linear boundary value problems. Meanwhile comparison were made between He's variational iteration method and ADM. 


\section{REFERENCES}

[1] M. A. Abdou and A. A. Soliman, Variational iteration method for solving Burger's and coupled Burger's equations, J. Comput. Appl. Math., 181 (2005) 245-251.

[2] M. A. Abdou, A. A. Soliman, New applications of variational iteration method, Physica D, $211(2005) 18$.

[3] G. Adomian, Solving Frontier Problems of Physics: The Decomposition Method, Kluwer, 1994.

[4] A. Borhanifar, H. Jafari and S. A. Karimi, New solitions and periodic solutions for the Kadomtsev-Petviashvili equation, J. Nonlinear Sci. Appl. 1 (2008), no.4, 224-229.

[5] J. H. He, Variational iteration method -Some recent results and new interpretations, J. Comput. Appl. Math., 207 (1) (2007) 3-17.

[6] J. H. He, Variational principle for some nonlinear partial differential equations with variable coefficients, Chaos, Solitons and Fractals, 19 (2004) 847-851.

[7] J. H. He, Variational iteration method for autonomous ordinary differential systems, Appl. Math. Comput., 114 (2000) 115-123.

[8] J. H. He, Variational principles for some nonlinear partial differential equations with variable coefficients, Chaos, Solitons and Fractals, 19 (2004) 847-851.

[9] J. H. He, Some asymptotic methods for strongly nonlinear equations, Int. J. Mod. Phys. B, 20 (10) (2006) 1141-1199.

[10] J. H. He, A new approach to nonlinear partial differential equation, Commun. Nonlinear Sci. Numer. Simulation 2 (1997) 230.

[11] M. Inokuti, et al., General use of the Lagrange multiplier in non-linear mathematical physics, in: S. Nemat-Nasser (Ed.), Variational Method in the Mechanics of Solids, Pergamon Press, Oxford, 1978, pp. 156-162.

[12] S. Momani and S. Abuasad, Application of He's variational iteration method to Helmholtz equation, Chaocs, Solitons and Fractals, 27 (2006) 1119-1123.

[13] A. M. A. Sayed, H. H. Ghashem, Solvability of nonlinear Hamerstein quadratic integral equations, J. Nonlinear Sci. Appl. 2 (2009), no.3, 152-160.

[14] A. M. Wazwaz, The variational iteration method for rational solutions for KdV, $\mathrm{K}(2,2)$,Burgers, and cubic Boussinesq equations, J. Comput. Appl. Math., 207(1) (2007) $18-23$.

[15] A. M. Wazwaz, A new algorithm for calculating Adomian polynomials for nonlinear operator, Applied Mathematics and Computation, 111 (2000), 5369.

[16] G. L. Liu, Weighted residual decomposition method in nonlinear applied mathematics, in: Proceedings of 6th Congress of Modern Mathematical and Mechanics, Suzhou, China, 1995, p. 643-648 (in Chinese).

[17] A. M. Wazwaz, A note on using Adomian decomposition method for solving boundary value problems, Foundations of Physics Letters, 13(5) (2000) 493-498.

[18] L. Yao, J. R. Chang, Variational principles for nonlinear Schrodinger equation with high nonlinearity, J. Nonlinear Sci. Appl. 1 (2008), no.1,1-4.

* School of Mathematics, Iran University of Science and Technology, NarMak, Tehran,Iran, P. O. BOX 16844.

E-mail address: golbabai@iust.ac.ir, sayehvand@iust.ac.ir 